\title{
Control of gear shifts in dual clutch transmission powertrains
}

\author{
*Paul D Walker ${ }^{1}$, Nong Zhang ${ }^{1}$, Richard Tamba ${ }^{2}$ \\ ${ }^{1}$ Faculty of Engineering and IT, University of Technology, Sydney \\ 15 Broadway, Ultimo, NSW, 2007, Australia \\ ${ }^{2}$ NTC Powertrains \\ Unit F, 2 Hudson Avenue, Castle Hill, 2154, Australia \\ *Corresponding author, Tel.: +61-2-9514-2987, fax: +61-2-9514 \\ email address: paul.d.walker@eng.uts.edu.au, \\ Postal address: \\ University of Technology, Sydney, \\ PO Box 123 \\ Broadway, NSW, Australia, 2007
}

\begin{abstract}
To achieve the best possible responses during shifting in dual clutch transmissions it is commonplace to integrate clutch and engine control, while the clutch is used to match speeds between the engine and wheels via reduction gears, poor engine control can lead to extended engagement times and rough/harsh shift transients. This paper proposes a method for combined speed and torque control of vehicle powertrains with dual clutch transmissions for both the engine and clutches. The vehicle powertrain is modelled as a simple four degree of freedom system with reduction gears and two clutches. Including a detailed clutch hydraulic model, comprising of the direct acting solenoids and clutch
\end{abstract}


piston with the hydraulic fluid modelled as a compressible fluid. Powertrain control is realised through control of clutch solenoids and manipulation of the engine throttle input. Sensitivity study of clutch performance evaluating inaccurate torque estimation demonstrated variance in the response of the hydraulic system, with an indicative simulation of poor estimation resulting in increased powertrain vibration during and after shifting. Simulations are conducted to demonstrate the capacity for this method of engine and clutch control to further reduce shift transients developed in dual clutch transmission powertrains. The obtained results also show that the adoption of torque based control techniques for both the clutch and engine, which makes use of the estimated target clutch torque, significantly improves the powertrain response as a result of reduction in the lockup discontinuities.

Keywords: Dual clutch transmission; Powertrain; Hydraulics; Control; Vibration;

\section{Introduction}

The combination of engine and clutch control in modern automated vehicular powertrains aims to deliver tractive driving torque to the road during shift transients in a manner providing high efficiency and excellent ride quality. While good system design can maximise these requirements for general driving, it is the engine and transmission control systems, including clutch hydraulics, which must provide ideal control of clutch and engine speeds and torques to achieve the best possible results during the shift period. This is particularly important for stepped transmission systems such as planetary 
automatics (AT) and dual clutch transmissions where control of both speed and torque control is required to minimise shift transients.

These transients are the result of three different discontinuities of speed, torque and inertia changes present in the powertrain during shifting. By minimising each of these three discontinuities the transient response of the powertrain can be reduced. Both the speed and torque of the engine and clutches can be controlled using different methods to satisfy the need for balancing of speed and torque. The nature of the DCT powertrain, however, requires the stepped change in inertia of the powertrain during shifting, which cannot be easily reduced without reducing the differences in consecutive gear ratios. The responses of a vehicle powertrain resulting from the control of both speed and torque are therefore the focus of this work, requiring detailed models of clutch hydraulics and powertrains for simulations.

Shift control in lightly damped or undamped powertrains such as the DCT requires the minimisation of transients that are capable of introducing undesirable vibrations. And for modern powertrains it requires the use of accurate torque and pressure sensors [1]. Comprehensive hydraulic system models presented in [2-4] have proven capable of providing accurate simulation of shift control for automated manual, continuously variable, and automatic transmissions, respectively. Detailed hydraulic models provide the link between control system and physical plant. With high nonlinearity and time delay existing in the hydraulics actuation system significantly increases the requirement for the development of detailed hydraulic models.

Similar methods have yet to be adopted in great detail for DCT shift control, Goetz [5-8] provides a number of examples of simulating shift control for DCTs, 
however only a simplified transfer function based on experimental data is used to model the hydraulic system. In the investigation of launch control [9], the proposed method simplifies the hydraulic control unit to a time delay to demonstrate the importance of gain selection for eliminating clutch judder in the DCT launch. These methods provide serious limitations in terms of control and accurate representation of hydraulic system response, particularly as the time delay and natural frequencies of the hydraulic system vary through the range of actuation of the hydraulic system. Other research reported in literature generally ignores the influence of the hydraulic control system on the shift control of DCTs [10-14], thus providing simulations of ideal actuation scenarios.

In studying the shift transients in automatic transmissions one popular method is to utilise multiple degree of freedom finite element models to investigate the dynamic behaviour of powertrains to shift transients. Crowther and et al [15] makes use of a four degree of freedom model for studying stick-slip in ATs, whilst for studying multiple clearances in the DCT 22 degrees of freedom are used [16]. Shim and Zhang [17] proposed a 14DOF model for the investigation of shifting quality on vehicle handling. It is, however, more common to use fewer degrees of freedom for shift control simulations $[18,19]$, e.g., a 3DOF state space model was employed for the development of speed based control of powertrains.

The purpose of this paper is to therefore demonstrate the role of integrated powertrain control of both engine and clutches in reducing shift transients for dual clutch transmissions with the inclusion of detailed hydraulic system models. Particularly, a special method is proposed to reduce the transient responses during the lock up of the clutch through adoption of a torque based control strategy of both clutches and engine. 
The presented layout of this paper is organised as follows. The development of the powertrain and hydraulic models is presented in the next section, with the inclusion of hydraulic system sensitivity analysis. Followed by the development and discussion of the control methodology, then simulations of typical shift transients are presented. Finally the findings and remarks are concluded regarding the modelling and simulation results.

\section{Model development}

Shift dynamics and control of stepped automatic powertrains has been demonstrated by [18] using speed based control of engine and clutch slip. Such methods have proved successful for automatic transmission with a 2-3DOF model. However the DCT requires more detail in responses and a 3-4DOF model will be developed. For DCT simulations the number of DOF utilised by different researchers changes significantly [12-14], but many researchers used 4DOF open clutch models for their work. In this section the simplified finite element based model of the DCT powertrain is developed, including the 3DOF closed clutch and 4DOF open clutch models, and the stick-slip clutch model and vehicle resistance torque.

\subsection{Lumped inertia model}

The development of a reasonable 4DOF finite element model of the dual clutch transmission powertrain uses elements provided for the engine, clutch drum, transmission, and vehicle. This is sufficient to identify the first three natural modes of the closed system, including one rigid body mode. The simplified powertrain is schematically shown in Figure 1 with clutches in the slip state, consistent with both clutches energised 
during general shifting conditions. The engine inertia takes input in the form of engine torque and outputs it to the clutch drum via shaft stiffness and damping element. The clutches are coupled to drum so there are two possible torques available as inputs that transmitted from the engine and clutch drum, as well as two outputs that drive the gear set either separately or simultaneously. The transmission transfers the clutch output torques to the propeller shaft, where it drives the vehicle inertia which is subjected to various loads such as air drag and rolling resistance.

The equations of motion of the open model are:

$$
\begin{aligned}
& I_{E} \ddot{\theta}_{E}=K_{D}\left(\theta_{D}-\theta_{E}\right)+C_{D}\left(\dot{\theta}_{D}-\dot{\theta}_{E}\right)+T_{E} \\
& I_{D} \ddot{\theta}_{D}=-K_{D}\left(\theta_{D}-\theta_{E}\right)-C_{D}\left(\dot{\theta}_{D}-\dot{\theta}_{E}\right)-T_{C 1}-T_{C 2} \\
& I_{T} \ddot{\theta}_{T}=K_{T}\left(\theta_{V}-\theta_{T}\right)+C_{D}\left(\dot{\theta}_{V}-\dot{\theta}_{T}\right)+C_{T}\left(\dot{\theta}_{V}-\dot{\theta}_{T}\right)+\gamma_{1} T_{C 1}+\gamma_{2} T_{C 2} \\
& I_{V} \ddot{\theta}_{V}=-C_{T}\left(\dot{\theta}_{V}-\dot{\theta}_{T}\right)-K_{T}\left(\theta_{V}-\theta_{T}\right)-T_{V}
\end{aligned}
$$

Where $\theta$ and its two derivatives are the rotational displacement, velocity, and acceleration, respectively, $\gamma$ represents the gear ratio, $I$ is the inertia element, $C$ is damping coefficient, $K$ is stiffness coefficient, and T is torque. For subscripts $E$ represents engine, $D$ for clutch drum, $T$ for transmission, $V$ is the vehicle, $C$ is clutch, and 1 and 2 represents the two clutches and respective gears.

When either of the two clutches is locked the vehicle reverts to a three degree of freedom model where the closed clutch merges the inertia of the drum with the transmission via a reduction gear, this gear ratio is the combination of both transmission ratio and final drive ratio for this model. A closed clutch model is presented in Figure 2 for clutch 1 locked and clutch 2 open. From here the importance of the reduction gearing 
is evident, providing step change in the speed, inertia and torques required for shifting between two clutches in the single transmission element.

The equations of motion of the closed model are:

$$
\begin{aligned}
& I_{E} \ddot{\theta}_{E}=K_{D}\left(\gamma_{1} \theta_{T}-\theta_{E}\right)+C_{D}\left(\gamma_{1} \dot{\theta}_{T}-\dot{\theta}_{E}\right)+T_{E} \\
& \left(\gamma_{1}^{2} I_{D}+I_{T}\right) \ddot{\theta}_{D}=-K_{D} \gamma_{1}\left(\gamma_{1} \theta_{T}-\theta_{E}\right)-C_{D} \gamma_{1}\left(\gamma_{1} \dot{\theta}_{T}-\dot{\theta}_{E}\right)+K_{T}\left(\theta_{V}-\theta_{T}\right)+C_{T}\left(\dot{\theta}_{V}-\dot{\theta}_{T}\right)
\end{aligned}
$$

$$
I_{V} \ddot{\theta}_{V}=-C_{T}\left(\dot{\theta}_{V}-\dot{\theta}_{T}\right)-K_{T}\left(\theta_{V}-\theta_{T}\right)-T_{V}
$$

The vehicle powertrain parameters of inertia, damping, and stiffness are presented below in Table 1 below.

\subsubsection{Free vibration analysis}

Damped free vibration analysis can be used to determine the vehicle natural frequencies, modes, and damping ratios. This method requires the representation of the model in state space form, represented in [20], before application of the eigenvalue problem [21]. This is performed for the three possible states of the powertrain using third and fourth gear. That assumes that both clutches are open, third or fourth gear engaged. For the analysis equations 1 to 4 and 5 to 7 are arranged in matrix form, and the external applied torques are assumed to be zero for free vibration. The equations are then in matrix form, presented as:

$$
[\mathbf{I}]\} \ddot{\theta}\}+[\mathbf{C}]\{\dot{\theta}\}+[\mathbf{K}]\{\theta\}=\{0\}
$$

Where I, $\mathbf{C}, \mathbf{K}$, are the inertia, damping, and stiffness matrices, and $\theta$ and its two derivatives are vectors of the displacement, velocity, and acceleration. To perform damped free vibration analysis the system matrix can be taken from equation (8), and the 
application of the eigenvalue problem can be used to determine natural frequencies and damping ratio. The system matrix is:

$$
[\mathbf{A}]=\left[\begin{array}{cc}
\mathbf{0} & \overline{\mathbf{I}} \\
\mathbf{I}^{-1} \mathbf{C} & \mathbf{I}^{-1} \mathbf{K}
\end{array}\right]
$$

With A being the system matrix, and $\overline{\mathbf{I}}$ being the identity matrix. The natural frequencies, and damping ratios are then in Table 2 below.

With the clutches open there are effectively two rigid body modes as the two separate powertrain halves are not coupled. Further, each of the open natural frequencies are associated with the two separate bodies, the higher frequency for the engine and clutch drum with low stiffness and high inertia, and the lower frequency for the transmission and vehicle body. With the clutches closed, however, the damping ratios are essentially identical and the natural frequencies are reasonably close, consistent with the change in effective inertia experienced by the locked drum and transmission inertias coupled via reduction gear pairs.

\subsection{Clutch model}

The use of piecewise clutch models to account for transitions between dynamic and static frictions is performed by [15], where upon clutch synchronisation an algorithm estimates the clutch torque and compares to the static friction to determine if friction lock is successful. A similar model is adopted by [10], however the post lockup torque is not well defined. In Goetz [6] a transfer function model of the hydraulic system is utilised over a detailed model, citing issues with the discontinuous contact in the clutch as a limiting factor. In this paper a nonlinear spring contact model is used to overcome this issue, thus a fourth variable of piston displacement is introduced to the piecewise model 
of the clutch, where the return spring separates the plate contact and the clutch torque drops to zero, excluding a small viscous contact component.

The piecewise model of the clutch is defined as a combination of dynamic and static friction where the static friction is calculated as the average torque in the clutch, and limited to the static friction of the clutch. This is defined as follows:

$$
T_{C}= \begin{cases}0 & X<X_{0} \\ n \mu_{D} \frac{2}{3} \frac{r_{0}^{3}-r_{I}^{3}}{r_{0}^{2}-r_{I}^{2}} \times F_{A} & X \geq X_{0},|\Delta \dot{\theta}| \geq 0 * \\ T_{\text {avg }} & X \geq X_{0},|\Delta \dot{\theta}|<0^{*}, T_{\text {avg }}<T_{C, S} \\ n \mu_{S} \frac{2}{3} \frac{r_{0}^{3}-r_{I}^{3}}{r_{0}^{2}-r_{I}^{2}} \times F_{A} & X \geq X_{0},|\Delta \dot{\theta}|<0^{*}, T_{\text {avg }} \geq T_{C, S}\end{cases}
$$

Where, $n$ is the number of friction plates, $X$ is piston displacement and $X_{0}$ is the minimum displacement required for contact between friction plates, $\mu_{D}$ is dynamic friction, $\mu_{S}$ is static friction, $r_{0}$ and $r_{I}$ are the outside and inside diameters of the clutch plates, and $F_{A}$ is the pressure load on the clutch.

A relatively simple model of the coefficient of friction of the clutches is presented as having dynamic friction, $\mu_{D}$, a static friction, $\mu_{S}$, for an absolute clutch speed of approximately zero, or $0^{*}$. Here $0^{*}$ is an approximation of zero such that numerical error in calculations is eliminated without negatively affecting results, including the phenomena of stick-slip.

With the average torque, $\mathrm{T}_{\mathrm{avg}}$, being derived from the open clutch equations for each of the two engaged clutches as:

$$
\begin{aligned}
& T_{a v g}=\left(\left|T_{C D 1,2}\right|+\left|T_{C T 1,2}\right|\right) / 2 \\
& T_{C D 1,2}=-I_{D} \ddot{\theta}_{D}-K_{D}\left(\theta_{D}-\theta_{E}\right)-C_{D}\left(\dot{\theta}_{D}-\dot{\theta}_{E}\right)-T_{C 2,1}
\end{aligned}
$$




$$
T_{C T 1,2}=\left(-I_{T} \ddot{\theta}_{T}+K_{T}\left(\theta_{V}-\theta_{T}\right)+C_{D}\left(\dot{\theta}_{V}-\dot{\theta}_{T}\right)+C_{T}\left(\dot{\theta}_{V}-\dot{\theta}_{T}\right)+\gamma_{2,1} T_{C 2,1}\right) / \gamma_{1,2}
$$

where equations (12) and (13) are realised by rearranging equations (2) and (3), respectively. Determining the average torque for clutch 1 or clutch 2 is achieved by using the alternate subscripts of 1 and 2 in sequential order. The average torque is important for torque based control of dual clutch transmissions as it is the target for the engaging clutch control in torque based control applications.

\subsection{Vehicle resistance model}

The vehicle resistance torques includes rolling resistance, aerodynamic drag, and incline of the road. This is given as:

$$
\begin{aligned}
& T_{V}=\left(F_{\text {Incline }}+F_{\text {Aerodynamic }}+F_{\text {Rollingresistance }}\right) \times R_{W} \\
& T_{V}=\left(M_{v} g \sin (\varphi)+\frac{1}{2} \rho_{\text {air }} A_{v} C_{D} V^{2}+M_{v} g C_{T}\right) \times R_{W}
\end{aligned}
$$

Where $M_{v}$ is vehicle mass, $g$ is gravity, $\varphi$ is angle of inclination, $A_{v}$ is vehicle area, $C_{D}$ is drag coefficient, $V$ is vehicle speed, $\rho_{a i r}$ is air density, and $C_{T}$ tire friction coefficient.

\subsection{Hydraulic control system model}

In dual clutch transmission systems the clutch control must be both responsive and accurate. Higher time delays present in control models for automatic transmissions, such as those presented in [4] or [22] are not capable of meeting DCT controller requirements, largely due to pilot solenoids controlling flow control valves. The development of responsive, high flow, direct acting solenoids similar to that presented in [23] have been required to improve clutch control in the DCT. To model such solenoids 
it is possible to apply conventional fluid dynamic principles to the complex valve system with multiple interconnected control volumes, examples of these applications are found in [24] and [25], which are used for the modeling presented in this section.

Figure 4 represents a simplified version of the clutch hydraulic control system, including one clutch piston and a direct acting, high flow, variable force solenoid similar to the valve presented in [23]. Eliminated from the control system are the pump, various pressure control and safety valves that have minimal influence on clutch control. These can be ignored if the line input pressure is assumed to be constant and the flow available from the pump is sufficient to supply hydraulic fluid to the cylinder actuator under all operating conditions.

The detail is reserved for modelling the mechanics and fluid dynamics of the solenoid valve, where consideration of flow variation from orifices, compressibility of the hydraulic fluid, and spool position all interact to throttle the flow to the clutch piston.

The equation of motion of the spool valve includes the input signal in the form of magnetomotive force, $F_{M}$, pressures from the two feedback volumes, $F_{1}$ and $F_{2}$, spring resistances, and frictional damping.

$$
M \ddot{x}=F_{M}-F_{1}+F_{2}-C \dot{x}-K_{1} x+K_{2} x
$$

To model the fluid dynamics of the hydraulic system the solenoid must be divided into as series of individual control volumes. Generally, these volumes are the input, output and feedback damping volumes in valves, and for a clutch pack the control volume is the main fill volume in the piston. Then the flow rates into and out of control volumes must be calculated using orifice flow equation for sharp edged orifices, defined below: 


$$
Q_{O}=C_{D} \pi \frac{D^{2}}{4} \sqrt{P_{2}-P_{1}}
$$

Here $Q_{O}$ is the orifice flow rate, $C_{D}$ is the discharge coefficient, $D$ is the orifice diameter, $P_{1}$ and $P_{2}$ are the diameters before and after the orifice, respectively.

Very often the flow into a control volume includes leakage between the spool and housing. The leakage flow can be calculated in a similar manner to the orifice equation only the input area becomes the annular clearance between the spool and housing, and is derived thus:

$$
Q_{L}=C_{D} \pi D c_{r} \sqrt{P_{2}-P_{1}}
$$

Here $D$ is the major diameter of the spool, and $c_{r}$ is the clearance between spool and valve body.

Equation (16) provides the area for a throttling orifice, for valve ports is somewhat different. If the port is closed the effective area is used as Equation 16, however, for the open port the effective area is a conical surface that is made between the edge of the spool and housing for the port. This area is shown in section detail below in figure 6:

To be utilised in the model the clearance " $c_{r}$ " in equation (18) can be written as follows:

$$
Q_{P}=C_{D} \pi D \sqrt{\left(X^{2}+c_{r}^{2}\right)\left(P_{2}-P_{1}\right)}
$$

For this $X$ is shown in Figure 6 as the opening width of the spool to any particular port.

Particular to the feedback volumes and the clutch pack is the rate of change in volume with the spool or piston in motion. This rate is expressed in the system as a flow 
rate change as fluid is forced into or out of the control volume. This is simply determined as from the spool area and instantaneous velocity of the valve.

$$
Q_{V}=A_{P} \frac{d X}{d t}
$$

Where $Q_{V}$ is the flow rate resulting from change in control volume and $A_{P}$ is the cross section area of the spool. The last source of change in flow arrises from the fluid compressibility, with hydraulic systems such as the one under investigation operating under high pressures in the mega-Pascal range the compressibility of the fluid cannot be ignored. The bulk modulus can be significantly affected by the presence of air in the hydraulic system, for the moment it is acceptable to ignore the effects of air. The flow resulting from compression of the hydraulic fluid is derived accordingly:

$$
Q_{C}=\frac{V}{\beta} \cdot \frac{d P}{d t}
$$

Where $\beta$ is the compressibility of the hydraulic fluid, $P$ is the control volume pressure, $Q_{C}$ is the flow rate resulting from compressibility of the hydraulic fluid, and $V$ is the volume of any particular control volume. Equations 16 to 21 are combined to provide the net flow into or out of any control volume in a hydraulic system; through mass conservation it is assumed that:

$$
\sum Q=0
$$

Or

$$
\mathrm{Q}_{\mathrm{O}}+\mathrm{Q}_{\mathrm{P}}+\mathrm{Q}_{\mathrm{L}}+\mathrm{Q}_{\mathrm{V}}+\mathrm{Q}_{\mathrm{C}}=0
$$

Thus 


$$
\begin{aligned}
& \frac{V}{\beta} \cdot \frac{d P}{d t}+A_{P} \frac{d X}{d t}+C_{D} \pi D \sqrt{\left(X^{2}+c_{r}^{2}\right)\left(P_{2}-P_{1}\right)}+ \\
& +C_{D} \pi D c_{r} \sqrt{P_{2}-P_{1}}+C_{D} \pi \frac{D^{2}}{4} \sqrt{P_{2}-P_{1}}=0
\end{aligned}
$$

And, by re-arranging (22) to determine the rate of pressure change, which can then be solved to determine the transient pressure change. This will then be integrated numerically to determine the local pressure in specific control volumes. With the inclusion of variation in the control volume pressure is calculated as:

$P=\int \frac{\beta}{V_{0}+d V}\left(A_{P} \frac{d X}{d t}+C_{D} \pi D \sqrt{\left(X^{2}+c_{r}{ }^{2}\right)\left(P_{2}-P_{1}\right)}+C_{D} \pi D c_{r} \sqrt{P_{2}-P_{1}}+C_{D} \pi \frac{D^{2}}{4} \sqrt{P_{2}-P_{1}}\right) d t$

This results in a first order differential equation that can be used to solve for the pressure in any control volume. The generic equation shown above was repeated in various forms six times for each solenoid valve, and with the inclusion of the spool dynamics a total of eight differential equations are required to characterise a single valve, and if the clutch piston is included this moves up to eleven equations to control a single clutch during shifting.

\subsection{Sensitivity analysis of hydraulic control system}

To evaluate the response of the hydraulic system to disturbances sensitivity analysis of the response of the clutch pressure to variation in the estimated average torque. To do so the hydraulic system is isolated from the powertrain model and two average torques are assumed, at 250 and $350 \mathrm{Nm}$. Additionally a +/-10\% variance is applied to each of these torques and at $1 \%$ increments simulations of the pressure response is carried out to determine the response to this pressure variation. 
The sensitivity study results are presented in Figure 7, where, for both plots, the torque variance represents the variation in average torque estimation in from the nominal torques in the legend, while the torque resultant refers to the actual torque generated in the clutch using the hydraulic control system. For each case the torque curves are reasonably linear, this is as best as can be expected with hydraulic control systems which are considered to be highly nonlinear systems. The lower average torque of $250 \mathrm{Nm}$ provide fairly accurate clutch torques in response to the average torque variance. However at the higher torque of $350 \mathrm{Nm}$ the accuracy is reduced significantly, with the variation from ideal torque at the extremities of $+/-10 \%$ being $2.5 \%$ from the nominal value.

\section{Control system methodology}

Shift control of complex vehicle powertrains such as the DCT requires the combined control of engine and clutch to ensure the minimisation of shifting transients. It is important in clutch control in particular to simulate both the pre-shift processes and torque and inertia phase control. The torque phase of clutch control is essentially the handover between the two phases, where the pressure in the engaged clutch is reduced, and the pressure in the target clutch is increased in a manner that ensures smooth changing of torque load between the two clutches, minimising the loss of output torque.

The inertia phase occurs after the engaged clutch is released, it is where the slip speed of the target clutch is reduced, and ending with the synchronising of the target clutch and lockup in the new gear, [26] provides a brief description of these phases for reference. 
Figure 8 presents the logic for realising torque based shift control of the DCT powertrain. Inputs of engine and clutch slip speeds, clutch pressures, and engine torque are used to control the engagement of the transmission in conjunction with the estimated average torque. During the shift clutch 1 pressure is reduced to match the estimated torque and clutch 2 is pre-filled. Next torque handover occurs between clutches, where PCL2 is increased to match the required torque, and PCL1 reduced to ensure smooth output torque, here slip is initiated according to Equation (10). The inertia phase contributes the majority speed synchronisation between clutch and drum, with target clutch held at constant torque and the control of engine throttle input aids shifting.

Estimation of the average torque is performed using equations 11 to 13 . The target pressure of the hydraulic control is defined from this variable, it should be considered as the torque required for maintaining current vehicle acceleration. In Figure 9 the typical combined clutch torques as experienced by the DCT transmission is demonstrated. The solid line represents the locked transmission, and the dashed line represents that clutches energised and slipping during the shift transient. Significant characteristics present include the torque hole that results from the handover of torque between releasing and engaging clutches this discontinuity would be observed by the driver as a loss of acceleration.

\subsection{Ideal clutch control}

Assuming ideal actuators to exclude the influence of the hydraulic control system on shifting and use the average torque to minimise torque hole, the hydraulic control system is replaced with ideal clutch torques representing clutch closed, torque and inertia 
phases. In doing so the clutch 1 torque is ramped down to zero during the torque phase, whilst clutch 2 torque increases to the target torque and held constant during the inertia phase. When either clutch is locked its respective torque is held at $1000 \mathrm{Nm}$, representing the maximum static friction in the clutch.

\subsection{Engine model and control}

Internal combustion engine modelling and control is a reasonably well established field of research. Examples of engine speed and torque control include control for manual transmissions[27], the general engine model [28] incorporating control of spark advance, throttle, exhaust gas recirculation, and air and fuel mass, and use of sliding mode control [26]. Alternatively dynamic engine models use baseline torque and harmonics to simulate torque pulses, as demonstrated by [16]. For our control requirements the higher frequency pulses from an engine will have limited influence on the control of engine speed and can be excluded from the modelling. The engine model adopts a lookup table of speed and torque using throttle angle to control output torque.

Two alternative control methods are utilised in this paper, simple inertia phase control to aid speed matching in the clutch is a well established method for engine control in general. An alternative engine control method is suggested now to improve torque balance at clutch lockup. Here engine torque is maintained during the torque phase, and during the majority of the inertia phase the engine speed is controlled by the throttle angle. As the target clutch approached synchronisation, the engine throttle angle is manipulated to match the required engine torque with the clutch. Thus, for the combined engine speed and torque control there will be matching of the required speeds and torques 
prior to clutch lockup, this is designed to minimise the discontinuities in the powertrain model and as a result reduce the system transients during clutch lockup.

\section{Simulation results and discussion}

For the simulations presented in this paper a general third to fourth gear up-shifts will be employed. Third gear ratio is 4.443 and fourth gear is 3.3912 , this includes the transmission reduction gear and final drive gears. The initial vehicle speed is approximately $70 \mathrm{rad} / \mathrm{s}$, equivalent to $80 \mathrm{~km} / \mathrm{h}$, resulting in initial engine speeds near 2800RPM. The final clutch torque is estimated using the average torque in Clutch 2 at the point of slip; this is similar to the torque profile in Figure 9. For this simulation the hydraulic control system is replaced by ideal input torques for the clutches, demonstrating the ideal response of the system.

The simulation results presented in Figure demonstrate the shift control of the DCT using the assumed ideal torque actuators discussed in section 3.1. Torques in Figure (c) represents the control signals to clutches. The torque phase is assumed to take $50 \mathrm{~ms}$ and a target torque of $300 \mathrm{Nm}$ is used for the maximum clutch 2 torque, and the overall duration of the transient is $350 \mathrm{~ms}$. The clutch 2 and drum speeds in figure 10 (a) are maintained during the torque phase as the torque on clutch 2 builds and clutch 1 is released, during the inertia phase clutch speed oscillates significantly with the end of torque phase, whilst post clutch lock up oscillations are present resulting from imbalance of engine torques and the unsteady transient slip speed generated in the clutch. vehicle acceleration in figure 10 (b) demonstrates a small drop in acceleration in the torque phase, representing the torque hole, and peak-to-peak post lockup oscillations are approximately 
$10 \mathrm{rads}^{-2}$, or with a wheel radius of $0.32 \mathrm{~m}$, a peak-to-peak variation of linear acceleration of $3.2 \mathrm{~ms}^{-2}$, a reasonable variation in acceleration given the simplification to a lumped mass model. The significance of light damping in the DCT compared to AT is also obvious with the damping of post lockup oscillations being very small.

The second set results are presented for a shift with identical initial conditions to the one detailed above, including torque control technique. To minimise the torque phase in this simulation using the detailed hydraulic system model the controlled torque phase is replaced by a step input for the target torque, minimising delay of the hydraulic system. Results for clutch engagement and vehicle acceleration are shown below:

Figure 11 presents the transient results for the powertrain model using the average torque from Clutch 2 as the target torque for clutch engagement with the modelled hydraulic controller, simple engine control is used to aid speed matching between clutch and drum. Unlike with ideal controllers when shifting is initiated at $2 \mathrm{~s}$ shift preparation proceeds actual shifting, this is represented in the clutch pressure profile in figure 11 (c) at 2 s clutch 1 pressure drops markedly to the target pressure in preparation for the initiation of slip, and there is a small increase in clutch 2 pressure signifying prefilling of the piston. In accordance with the piecewise clutch model, there is no contact in the friction plates and no friction torque is produced. Preparation takes approximately $100 \mathrm{~ms}$ before the torque phase begins with rapid handover between the two clutches, lasting less than 50ms. There is demonstration of overlapping torque in the two clutches with an initial in the vehicle acceleration increase during the torque phase. Similar to the ideal results the shift transient period is approximately $350 \mathrm{~ms}$ if shift preparation is excluded. The short torque phase and transition to constant torque in the inertia phase results in the 
clutch pressure overshooting the target torque before settling to just below the target pressure.

Similar transient vibration in clutch and drum speed synchronisation is generated during the inertia phase in comparison to Figure 10. However, in place of the small torque hole generated using ideal results overlap of clutch slip generates a brief increase in vehicle acceleration, in attempting to minimise the torque hole undesirable overlap is briefly produced, best observed in the vehicle acceleration. This is a result of time delay presenting in the release of clutch 1 and clutch 2 pressure increasing rapidly in attempt to minimise the torque hole.

As the clutch pressure settles additional variation in the vehicle acceleration during the inertia phase is produced in addition to basic oscillations. The results demonstrate transients generated in the transmission speed during shifting as a result of releasing the clutch, and transmission and clutch speeds upon lockup of the transmission. The post lock up vibration experienced in the transmission, (a), is also reflected in the vehicle acceleration response, (b), during the post shift steady state. This is increased in comparison to transients in Figure, resulting from error between clutch actual pressure and target pressure.

Using the same simulation parameters as used in the two previous cases this next simulation utilises torque based clutch control to perform shifting combined with torque balancing of the engine to target torque in the final stages of the inertia phase.

The results shown in Figure 12 are reasonably similar to those of Figure in terms of overall dynamics of powertrain and clutches. The use of engine torque control at the end of engagement increases the duration of the inertia phase as additional energy is 
applied to the system, resisting the clutch torque engagement. In Figure 13 (a) the synchronisation is slowed in the final stages of the inertia phases as increased energy is imparted on the system, and the resulting post shift transients in the closed clutch is significantly reduced from Figure 11. The vehicle acceleration in part (b) of Figure 12 supports the improved post shift transient vibration, with peak-to-peak vibrations smaller than those presented in all previous simulations, again there is evidence of clutch overlap in the torque phase of shifting. From part (c), the pre-shift clutch release and pre-fill is identical to the previous simulation. The torque phase is the same, but the inertia phase is extended as a result of employing the engine torque control. The overall shift transient period, shown in part (c) is extended, taking over $400 \mathrm{~ms}$, also a result of the new engine control method. The vehicle speed, as shown in part (b), demonstrates minimal loss in acceleration and tractive load to the road resulting from the clutch-on-clutch shifting. In particular transients developed in the transmission and vehicle responses are significantly reduced in comparison to Figure. With the only change to the control algorithm being the torque balancing in the engine in the final stages of the inertia phase, it is possible to conclude that significant improvements can be made by balancing system torques prior or at the completion of clutch lockup to minimise discontinuities in the system torques.

To further demonstrate the results of inaccurate estimation of the target torque and the impact on gear shifting the estimated clutch two torque is increased to $110 \%$ of the ideal torque. Immediately the results in Figure 13 show degradation of performance in comparison to both figures 11 and 12. Figure 13 (a) indicates increased vibration during the inertia phase as well as amplified amplitudes during post shift vibration. Additionally, figure 13 (b) indicates enhanced torque overlap during the torque phase, noting that slip 
begins at approximately the same time in each of the simulations, and vehicle acceleration oscillations is significantly increased also. Thus the detrimental effects of inaccurate torque estimation are clearly demonstrated in the simulations.

\section{Conclusions}

The development of speed and torque orientated shift control methods for dual clutch transmissions have been presented in this paper. This includes a 4DOF finite element model of the vehicle powertrain and a detailed model of the clutch hydraulics using classical fluid dynamics. The detailed model of the clutch hydraulic system is required to demonstrate realistic responses of clutch control during shift transients. Control methodologies focus on the integration of clutch torque, engine speed and torque control techniques. A sensitivity study was performed for evaluating the variation of torque developed at the clutch in comparison to the average torque estimated by the controller. Demonstrating that there is some inaccuracy for clutch torque resulting from the hydraulic system as torque diverges from nominal parameters at higher input torques. Simulations using values exceeding nominal were shown to increase vibration in the system above nominal results.

The use of ideal torque parameters indicated that it is possible to minimise the torque hole using optimal torque profiles. The capability for this to be transferred to the detailed hydraulic system model, though, is somewhat limited, with a small degree of overlapping torque increasing mean acceleration of the vehicle during the torque phase. The use of nominal estimated torques during the shifting with and without torque control of the engine, used to balance input to output torques immediately prior to clutch lockup 
also demonstrated reduction in torsional oscillations in the system. However, this method also resulted in the extension of the transient period during shifting. These results indicate that the adoption of feed forward control of engine torque has the potential to reduce the DCT transient response during shifting.

\section{Acknowledgements}

The financial support of this work by the Australian Research Council (ARC LP0775445) and the University of Technology, Sydney, is gratefully acknowledged.

\section{References}

[1] Z. Sun, K. Hebbale, Challenges and opportunities in automotive transmission control, in: American control conference, Portland, USA, 2005, pp. 3284-3289.

[2] G. Lucente, M. Montanari, C. Rossi, Modelling of an automated manual transmission system, Mechatronics, 17 (2007), 73-91.

[3] M. Pesgens, B. Vroemen, B. Stouten, F. Veldpaus, M. Steinbuch, Control of a hydraulically actuated continuously variable transmission, Vehicle System Dynamics, 44 (2006), 387-406.

[4] J. Jeyakumaran, N. Zhang, Friction induced vibration of clutches in an automatic transmission, Proceedings of the Asia Pacific Vibration Conference, Langkawi, Malaysia, 2005, pp. 174-179.

[5] M. Goetz, Integrated Powertrain control for Twin Clutch Transmissions, Ph.D. Thesis, School of Mechanical Engineering, University of Leeds, Leeds, Great Britain, February 2005. 
[6] M. Goetz, M. Levesley, D. Crolla, Dynamics and Control of Gearshifts on TwinClutch Transmission, Proceedings of the institution of mechanical engineers, Part D: Journal of Automobile Engineering, 219 (2005), 951-963.

[7] M. Goetz, M. Levesley D. Crolla, Dynamic Modelling of a Twin Clutch Transmission for Crontroller Design, Materials Science Forum, 440-441 (2003), 253-261.

[8] M. Goetz, M. Levesley, D. Crolla, A Gearshift Controller for Twin Clutch Transmissions, VDI Berichte, 1786 (2003), pp. 381-400.

[9] S. Kirschstein, The Impact of Launch Control on the Vibration Behaviour of a Dual Clutch Transmission Powertrain, VDI Berichte, 1971 (2007), 197-217.

[10] M. Kulkarni, T. Shim, Y. Zhang, Shift Dynamics and Control of Dual-Clutch Transmissions, Mechanism and Machine Theory, 42 (2007), 168-182.

[11] X. Song, J. Liu, D. Smedley, Simulation Study of Dual Clutch Transmission for Medium Duty Truck Application, SAE Technical Paper 2005-01-3590, (2005).

[12] G. Xuexun, F. Chang, Y. Jun, Y. Zheng, Modelling and Simulation Research of Dual Clutch Transmission Based on Fuzzy Logic Control, SAE Technical Paper 200701-3754, (2007).

[13] Y. Zhang, X. Chen, X. Zhang, H. Jiang, W. Tobler, Dynamic Modelling and Simulation of a Dual-Clutch Automated Lay-Shaft Transmission, Transactions of the American Society for Mechanical Engineers, Journal of Mechanical Design, 127 (2005), 302-307.

[14] Y. Lei, J. Wang and A. Ge, "Research on control stratgies of double clutch transmission based on system simulation," in: Proceedings of FISITA World Automotive Congress, Yokohama, Japan, October 2006, Paper number F2006P041. 
[15] A. Crowther, N. Zhang, D. K. Liu, J. Jeyakumaran, Analysis and Simulation of Clutch Engagement Judder and Stick-Slip in Automotive Powertrain Systems, Proceedings of the Institution of Mechanical Engineers, Part D: Journal of Automobile Engineering, 218 (2004), 1427-1446.

[16] A. R. Crowther, R. Singh, N. Zhang, C. Chapman, Impulsive response of an automated transmission system with multiple clearances: formulation, simulation, and experiment, Journal of Sound and Vibration, 306 (2007), 444-466.

[17] T. Shim, Y. Zhang, Effects of Transient Powertrain Shift Dynamics on Vehicle Handling, Int. J. Vehicle Design, 40 (2006), 159-174.

[18] U. Kiencke, L. Nielson, Automotive Control Systems, Springer, Germany, 2005.

[19] A. Serrarens, M. Dassen, M. Steinbuch, Simulation and control of an automotive dry clutch, in: Proceedings of the American Control Conference, Boston, USA, 2004, pp. 4078-4083.

[20] K. Ogata, Modern Control Engineering, Prentice Hall, USA, 1997.

[21] S. S. Rao, Mechanical Vibrations. Addison-Wesley Publishing, USA, 1986,

[22] S. Watechagit, K. Srinivasan, Modelling and Simulation of Shift Hydraulic System for a Stepped Automatic Transmission, SAE Technical Paper, 2003-01-0314, (2003).

[23] G. R. Holmes and R. Tamba, "Solenoid control valve," Patent United States of America US 7,082,965 B2, 1 August 2006.

[24] N. D. Manring, Hydraulic Control Systems, John Wiley \& Sons, USA, 2005.

[25] J. Stringer, Hydraulic Systems Analysis, an Introduction, Macmillan Press Ltd, London, UK, 1976. 
[26] J. J. Moskwa, J. K. Hedrick, Nonlinear algorithms for Automotive Engine Control, IEEE Control Systems Magazine, (1990), pp. 88-92.

[27] F. Amisano, G. Serra, M. Velardocchia, Engine control strategy to optimise a shift transient during clutch engagement, SAE Technical Paper, 2001-01-0877, (2001).

[28] P. R. Crossley and J. A. Cook, "A nonlinear engine model for drivetrain system development," in: IEEE International Conference on Control, Edinburgh, Scotland, 1991, pp. 921-925. 


\section{List of Figures}

Figure 1: 4DOF model of DCT powertrain with both open clutches

Figure 2: 3DOF dynamic model of powertrain with clutch one closed

Figure 3: Clutch friction model

Figure 4: Simplified hydraulic system for clutch control

Figure 5: Typical solenoid valve section based on [23]

Figure 6: Detail of flow area for open port

Figure 7: Sensitivity analysis results, (left) torque resultant to average torque estimation, (right) Percent torque resultant to torque estimation

Figure 8: Control logic for gear shift of DCT powertrains

Figure 9: Typical torque response during shifting

Figure 10: Simulation results for a 3-4 up-shift showing speeds for (a) Clutch drum and clutch 2, (b) vehicle acceleration, and (c) clutch pressures during shifting using ideal clutch torques

Figure 11: Simulation results for a 3-4 up-shift with minimised time delay showing speeds for (a) clutch drum and clutch 2, (b) vehicle acceleration, and (c) clutch pressures during shifting using engine speed control and Clutch 2 average torque estimation Figure 12: Simulation results for a 3-4 up-shift showing speeds for (a) clutch drum and clutch, (b) vehicle acceleration and (c) clutch pressures during shifting using engine speed and torque control, and clutch 2 average torque estimation 
Figure 13: Simulation results for a 3-4 up-shift with variance in estimated torque for controller input showing speeds for (a) clutch drum and clutch, (b) vehicle acceleration, and (c) clutch pressures 


\section{Tables}

Table 1: Vehicle powertrain parameters

\begin{tabular}{llll}
\hline Inertia & $\left(\mathbf{k g} / \mathbf{m}^{\wedge} \mathbf{2}\right)$ & Stiffness elements & $(\mathbf{N m} / \mathbf{r a d})$ \\
\hline $\mathbf{I}_{\mathbf{E}}$ & 0.6 & $\mathbf{K}_{\mathbf{D}}$ & 77000 \\
$\mathbf{I}_{\mathbf{D}}$ & 0.2 & $\mathbf{K}_{\mathbf{T}}$ & 16000 \\
\cline { 3 - 3 } & 0.28 & Damping elements & $(\mathbf{N m . s} / \mathbf{r a d})$ \\
$\mathbf{I}_{\mathbf{T}}$ & 68.82 & $\mathbf{C}_{\mathbf{D}}$ & 1.76 \\
- & - & $\mathbf{C}_{\mathbf{T}}$ & 0.12 \\
\hline
\end{tabular}

Table 2: vehicle natural frequencies and damping ratios

\begin{tabular}{|c|c|c|c|c|c|c|c|c|c|c|}
\hline & \multicolumn{4}{|c|}{ Clutches open } & \multicolumn{3}{|c|}{$3^{\text {rd }}$ gear engaged } & \multicolumn{3}{|c|}{$4^{\text {th }}$ gear engaged } \\
\hline Natural & 0 & 0 & 38 & 114 & 0 & 14 & 98 & 0 & 17 & 91 \\
\hline \multicolumn{11}{|c|}{ frequencies } \\
\hline \multicolumn{11}{|l|}{$(\mathbf{H z})$} \\
\hline Damping & - & - & 0.01 & 0.01 & - & 0.2 & 0.3 & - & 0.2 & 0.3 \\
\hline ratio $(\%)$ & & & & & & & & & & \\
\hline
\end{tabular}

